\title{
Lactobacillus gasseri: effects on mouse intestinal flora enzyme activity and isoflavonoids in the caecum and plasma
}

\author{
Motoi Tamura*, Mayumi Ohnishi-Kameyama and Kazuki Shinohara \\ National Food Research Institute, Tsukuba 305-8642, Japan
}

(Received 27 April 2004 - Revised 23 July 2004 - Accepted 3 August 2004)

\begin{abstract}
The effects of Lactobacillus gasseri JCM $1131^{\mathrm{T}}$ on isoflavonoid levels within the caecum and plasma were assessed in adult mice. Male 5-week-old mice were fed an AIN 93M diet for $30 \mathrm{~d}$. Two groups of mice were administered either L. gasseri JCM $1131^{\mathrm{T}}$ (the LGI group) or physiological saline solution (the control (CI) group) daily for $5 \mathrm{~d}$ before dissection. The plasma daidzein concentration was significantly higher in the LGI group, however, their plasma equol concentration was significantly less than in the CI group. The total amount of equol present as aglycone in the caecum was significantly greater in the CI group, but there was no significant difference in the total daidzein present as caecal aglycone. In an in vitro incubation of daidzin with the faecal flora of mice, the equol concentration was significantly higher in the CI group. The numbers of lactobacilli present were significantly higher in the LGI group. The present data suggest that the administration of L. gasseri is likely to influence the effect of isoflavonoids on the host via changes in the gastrointestinal environment.
\end{abstract}

Daidzein: Equol: Lactobacillus gasseri: Caecal enzyme activity

Phyto-oestrogens are defined as compounds that exert oestrogenic effects on the central nervous system, induce oestrus, and stimulate growth of the genital tract in female animals (Lieberman, 1996). Plant phyto-oestrogens include the soya isoflavones, which in addition to their oestrogenic effects may be partially responsible for the protective effects of soya against CVD (Anthony et al. 1998). Daidzin is a major component of these isoflavones. Daidzin is the 7-glucoside form of daidzein. Daidzein is metabolised to equol by the intestinal bacterial flora (Bowey et al. 2003). Equol is considerably more oestrogenic than daidzein (Shutt \& Cox, 1972).

The intestinal flora plays a key role in the metabolism and bioavailability of isoflavones (Setchell et al. 1984). As such, infants fed soya-containing infant formula in the first 4 months of life (when gut microflora are underdeveloped) cannot produce large quantities of equol (Cruz et al. 1994; Setchell et al. 1997). Furthermore, only about $30-40 \%$ of adult subjects excrete significant quantities of equol after isoflavone consumption (Setchell et al. 1984; Lampe et al. 1998). Probiotics have been defined as live microbial food supplements which benefit the recipient host by improving the intestinal microbial balance (Fuller, 1989). Lactic acid bacteria have been used as probiotics with the aim of managing intestinal disorders such as lactose intolerance, acute rotavirus gastroenteritis (and other enteric pathogens), the adverse effects of pelvic radiotherapy, constipation, inflammatory bowel disease and food allergy (Salminen et al. 1988, 1996; Gilliland, 1990; Isolauri et al. 1991; Hammes \& Tichaczek, 1994).

Lactobacillus gasseri, in the L. acidophilus group, is a major species of the human faecal flora. It has been reported that the $L$. gasseri strain, isolated from infant faeces, shows wide inhibitory activity against some pathogenic and food-spoilage species, including Clostridium, Listeria and Enterococcus (Zhu et al. 2000). L. gasseri may affect the intestinal flora and hence the metabolism of isoflavonoids within the digestive tract. However, there are few reports of the effects of L. gasseri on caecal and plasma isoflavonoids, or on caecal enzyme activity. Therefore, the aim of the present study was to investigate these effects in mice.

\section{Materials and methods}

Materials

A soyabean extract with a high isoflavone-glucoside content was kindly provided by Fuji Oil Co., Ltd. (Osaka, Japan). It contained $31.1 \%(\mathrm{w} / \mathrm{w})$ daidzin, $9.6 \%(\mathrm{w} / \mathrm{w})$ genistin and $38.5 \%(\mathrm{w} / \mathrm{w})$ gycitin. Daidzein used as a standard for HPLC analysis was purchased from Fujicco (Kobe, Japan), as was daidzin. Equol was purchased from Extrasynthese (Genay, France). $\beta$-Glucuronidase type $\mathrm{H}-5, p$-nitrophenyl- $\beta$-D-glucopyranoside and $p$-nitrophenyl- $\beta$-D-glucuronide were obtained from Sigma, 
Aldrich Corp. (St Louis, MO, USA). L. gasseri JCM 1131 ${ }^{\mathrm{T}}$ (type strain) was obtained from the Institute of Physical and Chemical Research (RIKEN, Wako-shi, Saitama, Japan).

\section{Treatment of animals with Lactobacillus gasseri}

Male Crj: CD-1 (ICR) mice (5 weeks old) were purchased from Charles River Japan Inc. (Kanagawa, Japan). The animals were housed in suspended stainless-steel cages with wire-mesh bottoms in a room kept at $24 \pm 0.5^{\circ} \mathrm{C}$ and a relative humidity of $65 \%$, with alternating $12 \mathrm{~h}$ periods of light and dark. They were fed an AIN-93M purified diet (Reeves et al. 1993) for $30 \mathrm{~d}$. The L. gasseri JCM $1131^{\mathrm{T}}$ type strain was incubated on EG agar (Nissui Pharmaceutical Co. Ltd., Tokyo, Japan) for $2 \mathrm{~d}$ at $37^{\circ} \mathrm{C}$ in an anaerobic atmosphere generated using an AnaeroPack ${ }^{\circledR}$ (Mitsubishi Gas Chemical Company Inc., Tokyo, Japan). An inoculating loop of the bacteria was then suspended in $2 \mathrm{ml}$ physiological saline solution.

The mice were randomly divided into two groups of seven animals each. The mice had been fed the AIN $93 \mathrm{M}$ diet for $30 \mathrm{~d}$. One group received L. gasseri (the L. gasseri group; LGI group) and one did not (control group; CI group). A $0.2 \mathrm{ml}$ dose of L. gasseri $\left(10^{9}\right.$ colony-forming units $/ 0.2 \mathrm{ml}$ ) was administered via a stomach tube to the LGI group of mice for $5 \mathrm{~d}$. The mice in the CI group were administered $0.2 \mathrm{ml}$ physiological saline solution via a stomach tube, under the same dietary and living conditions.

At $5 \mathrm{~d}$ before the administration of $L$. gasseri (or physiological saline), mouse faeces were collected and immediately processed for bacteriology. On the fifth day after the administration of L. gasseri (or physiological saline) mouse faeces were again collected and a faecal incubation test with daidzin was performed. During the night before dissection, a soyabean extract with a high isoflavone-glucoside content $(136 \mathrm{mg} ; 31.1 \%(\mathrm{w} / \mathrm{w})$ daidzin, $9.6 \%$ (w/w) genistin and $38.5 \%(\mathrm{w} / \mathrm{w})$ glycitin) was freshly prepared as a $3 \mathrm{ml}$ suspension in water. A $0.2 \mathrm{ml}$ dose was administered to the mouse via a stomach tube. At $12 \mathrm{~h}$ later, all mice were killed and dissected. The mice had unrestricted access to food throughout the experiment. The mice were then anaesthetised with diethyl ether and blood samples were taken from the abdominal aorta and placed in heparinised tubes. The plasma was separated from whole blood by centrifugation and stored at $-80^{\circ} \mathrm{C}$ before HPLC analysis for isoflavonoids. Caecal contents were also collected and stored at $-80^{\circ} \mathrm{C}$ until caecal enzyme activity and caecal isoflavonoids content were measured. The present study was carried out in accordance with the 'Guidelines for Animal Care and Experimentation' of the National Food Research Institute.

\section{Analysis of plasma and caecal isoflavonoids}

The analysis of plasma isoflavonoids was performed as follows. Plasma $(200 \mu \mathrm{l})$ was added to $200 \mu \mathrm{l} \beta$-glucuronidase type H-5 solution $(35 \mathrm{mg} / \mathrm{ml}$; Sigma-Aldrich Corp., St Louis, MO, USA) in $0.2 \mathrm{M}$-sodium acetate buffer ( $\mathrm{pH} 5 \cdot 0$ ). $\mathrm{Next}$, the mixture was incubated at $37^{\circ} \mathrm{C}$ in a shaking water-bath for $2 \mathrm{~h}$, followed by treatment with $3600 \mu \mathrm{l}$ methanol-acetic acid (100:5, v/v), vortexing for $30 \mathrm{~s}$, sonication for $30 \mathrm{~s}$, vortexing again for $30 \mathrm{~s}$ and centrifugation at $5000 \mathrm{~g}$ for $10 \mathrm{~min}$ at $4^{\circ} \mathrm{C}$. The supernatant fractions were transferred to an eggplant-type flask and evaporated completely using a rotary evaporator. The sample was then dissolved within the mobile phase of the HPLC system in a volume equivalent to the original plasma and filtrated through a $0.2 \mu \mathrm{m}$ filter. During HPLC analysis, $20 \mu \mathrm{l}$ of each preparation was injected into a $250 \times 4.6 \mathrm{~mm}$ Capcell Pak C18-5 $\mathrm{m}$ column (Shiseido, Tokyo, Japan). Isoflavonoids were detected using an ECD with a guard cell (model 5020), and an analytical cell (model 5010) (Coulochem II; ESA Inc., Bedford, MA, USA). The mobile phase consisted of methanol-acetic acid-water (28:5:67, by vol.). The running conditions for HPLC were as follows: column temperature, $40^{\circ} \mathrm{C}$; flow rate, $1 \mathrm{ml} / \mathrm{min}$; guard cell, $+850 \mathrm{mV}$; analytical cell, $+300 \mathrm{mV}$ for electrode 1 and $+800 \mathrm{mV}$ for electrode 2 . Electrochemical data were collected from electrode 2 . To perform the analysis of caecal isoflavonoid aglycones, caecal contents were treated with $2 \mathrm{ml}$ methanol-acetic acid (100:5, v/v), vortexed for $30 \mathrm{~s}$, sonicated for $30 \mathrm{~s}$, vortexed again for $30 \mathrm{~s}$, and centrifuged at $5000 \mathrm{~g}$ for $10 \mathrm{~min}$. The supernatant fraction was then transferred to the eggplant-type flask. The same volume as the first extraction of methanol-acetic acid (100:5, v/v) was added to the sediment, and the procedure was repeated. The supernatant fractions from both extractions were pooled and evaporated completely using a rotary evaporator. Then, the sample was dissolved within $3 \mathrm{ml}$ of the mobile phase of the HPLC system and filtrated through a $0.2 \mu \mathrm{m}$ filter. We used the same HPLC analytical method as described earlier.

\section{Measurement of enzyme activity}

We measured enzyme activities as previously described (Rowland et al. 1983). A 1:100 caecal suspension was prepared in $0 \cdot 1 \mathrm{M}$-phosphate buffer $(\mathrm{pH} \mathrm{7 \cdot 0)}$ and non-bacterial debris was removed by centrifugation at $700 \mathrm{~g}$ for $2 \mathrm{~min}$. The supernatant fraction was used immediately in the $\beta$-glucosidase and $\beta$-glucuronidase assays. $\beta$-Glucosidase activity was measured with $p$-nitrophenyl- $\beta$-D-glucopyranoside as the substrate. $\beta$-Glucuronidase activity was measured with $p$-nitrophenyl- $\beta$-D-glucuronide as the substrate. $\beta$-Glucosidase and $\beta$-glucuronidase activities were expressed as $\mu \mathrm{mol} p$-nitrophenol liberated/60 min per $\mathrm{g}$ wet weight caecal contents.

\section{Bacteriology}

Faeces were weighed, serial 10-fold dilutions were prepared and then homogenised using a sterile glass homogeniser. The diluted samples were spread on the surface of LBS agar media (Becton Dickinson and Company, Sparks, MD, USA) and incubated at $37^{\circ} \mathrm{C}$ in an anaerobic atmosphere generated using an AnaeroPack ${ }^{\circledR}$ (Mitsubishi Gas Chemical Company Inc., Tokyo, Japan). Bacteria were identified to the level of genus on the basis of colony form, Gram stain and cell morphology. Bacterial numbers were expressed as $\log _{10}$ colony-forming units/g wet weight faeces. 
In vitro incubation of daidzin with faecal flora of mice

Freshly voided mouse faeces were collected in sterile glass homogenizers. Fifty volumes of the anaerobic broth were added to the faeces, and the mixture was homogenized. Daidzin $(20 \mathrm{mg})$ was dissolved in $1 \mathrm{ml}$ dimethyl sulfoxide. Daidzin solution $(3 \mu \mathrm{l})$ was transferred into $0.3 \mathrm{ml}$ homogenate then incubated anaerobically at $37^{\circ} \mathrm{C}$ for $24 \mathrm{~h}$. After incubation, the reaction mixture was treated with nine volumes of methanol-acetic acid (100:5, v/v). The mixture was vortexed for $30 \mathrm{~s}$, sonicated for $30 \mathrm{~s}$, vortexed again for $30 \mathrm{~s}$ and centrifuged at $5000 \mathrm{~g}$ for $10 \mathrm{~min}$. The supernatant fraction was transferred to a test-tube and filtered through a $0.2 \mu \mathrm{m}$ filter. HPLC analysis was performed by injecting $20 \mu \mathrm{l}$ of each preparation into a $250 \times 4.6 \mathrm{~mm}$ Capcell Pak C18-5 $\mu \mathrm{m}$ column (Shiseido, Tokyo, Japan). The anaerobic broth used in this experiment was prepared as follows. Brain-heart infusion (37 g), agar $(1 \mathrm{~g})$, L-cysteine $\mathrm{HCl}(0.5 \mathrm{~g})$, and $\mathrm{Na}_{2} \mathrm{CO}_{3}(4 \mathrm{~g})$ were dissolved in $1000 \mathrm{ml}$ distilled water. Samples of broth $(9 \mathrm{ml})$ were distributed into test-tubes, gassed with $\mathrm{O}_{2}$-free $\mathrm{CO}_{2}$ gas, sealed with a butyl rubber stopper, and sterilised by autoclaving. HPLC analysis was performed as described earlier.

\section{Statistics}

The data are expressed as mean values with their standard errors. Data were analysed using SigmaStat for windows (Jandel Corporation, San Rafael, CA, USA) and $t$ test analysis.

\section{Results}

\section{Body weight, food consumption, and caecal contents}

No significant differences in final body weight $(\mathrm{g})$ were observed between the LGI (38.4 (SE 0.9)) and the CI (37.3 (SE 1.0)) groups. No significant differences in food consumption $(\mathrm{g} / \mathrm{d})$ were observed between the LGI $(4.8$ (SE 0.1)) and the CI (4.8 (SE 0.1)) groups. There were no significant differences in the caecal contents between the LGI and CI groups.

\section{Plasma isoflavonoids and caecal isoflavonoid aglycones}

The plasma daidzein concentration was significantly higher in the LGI group compared with the CI group (Fig. 1). Conversely, the plasma equol concentration was significantly lower in the LGI group (Fig. 1). There was a significant difference in the plasma equol:plasma daidzein ratio between the LGI and CI groups. The plasma equol:plasma daidzein ratio in the LGI group (4.3 (SE 2.6)) was significantly lower compared with the CI group (19.7 (SE 4.9)) $(P<0.05)$. The total amount of equol present as aglycone in the caecum was significantly higher in the CI group compared with the LGI group (Fig. 2). However, there was no significant difference in the total amount of daidzein present as aglycone in the caecum (Fig. 2). The ratio of the total amount of daidzein present as aglycone in the caecum to the total amounts of equol present as aglycone in the caecum tended to be high in the CI

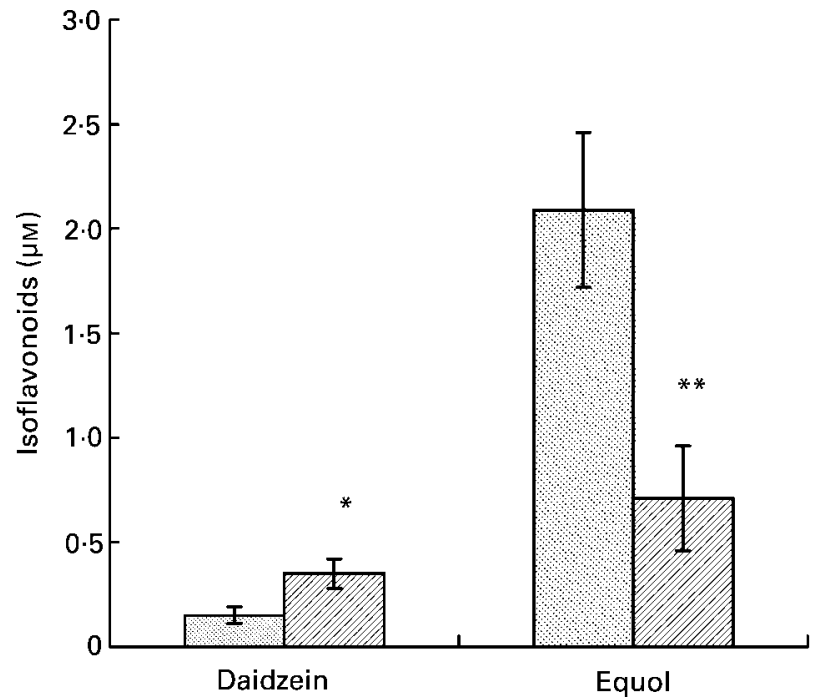

Fig. 1. Plasma isoflavonoids (aglycones + metabolites) of the mice in the control group (图) and the Lactobacillus gasseri (LGI) group $(\mathbb{Q})$. Values are means, with their standard errors represented by vertical bars. *Mean value was significantly different from that for the $\mathrm{Cl}$ group $(P<0.05)$. ${ }^{* *}$ Mean value was significantly different from that for the $\mathrm{Cl}$ group $(P<0.01)$.

group (6.3 (SE 3.5)) compared with the LGI group (1.1 (SE 0.5)). However, no significant difference was observed in these ratios between the LGI and CI groups $(P=0 \cdot 158)$.

\section{Caecal enzyme activity}

No significant differences in caecal $\beta$-glucosidase and $\beta$-glucuronidase activities were detected between the LGI and CI groups (Fig. 3).

\section{Measurement of numbers of lactobacilli}

Significant differences were observed in the numbers of lactobacilli between the two groups. There were significantly higher numbers in the LGI group (Fig. 4).

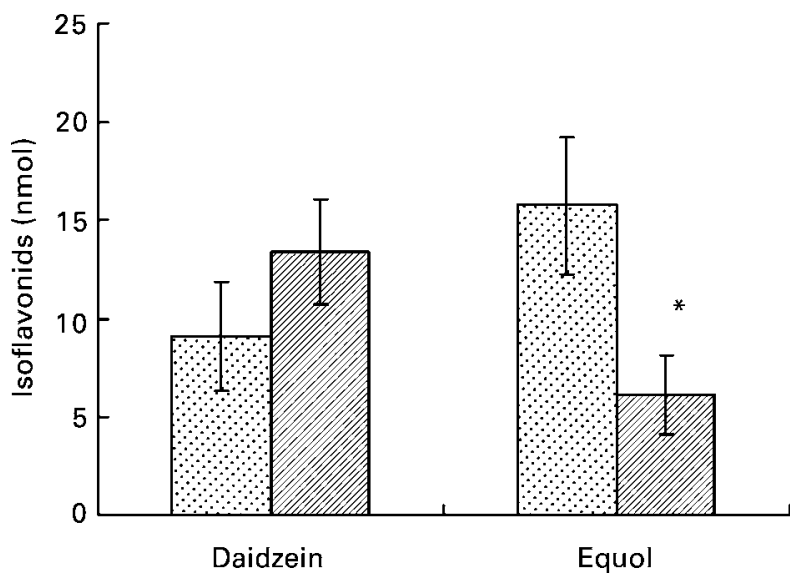

Fig. 2. Total amounts of isoflavonoids present as aglycone in the caecum of the mice in the control group (圈) and the Lactobacillus gasseri group (四). Values are means, with their standard errors represented by vertical bars. *Mean value was significantly different from that for the $\mathrm{Cl}$ group $(P<0.05)$. 


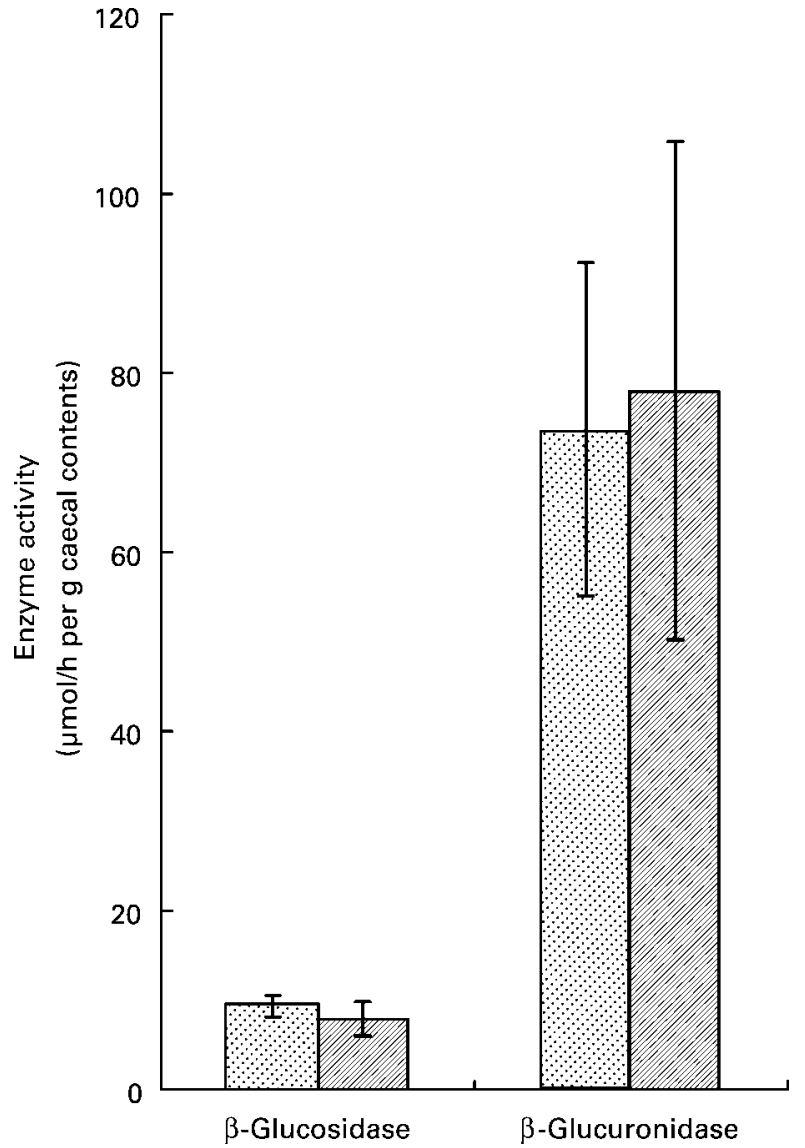

Fig. 3. Caecal $\beta$-glucosidase and $\beta$-glucuronidase activities of the

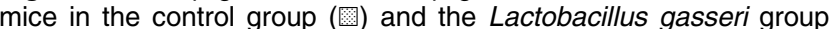
(ख). Values are means, with their standard errors represented by vertical bars.

In vitro incubation of daidzin with the faecal flora of mice

There was a significant difference in equol concentration between the two groups, with the CI group having a

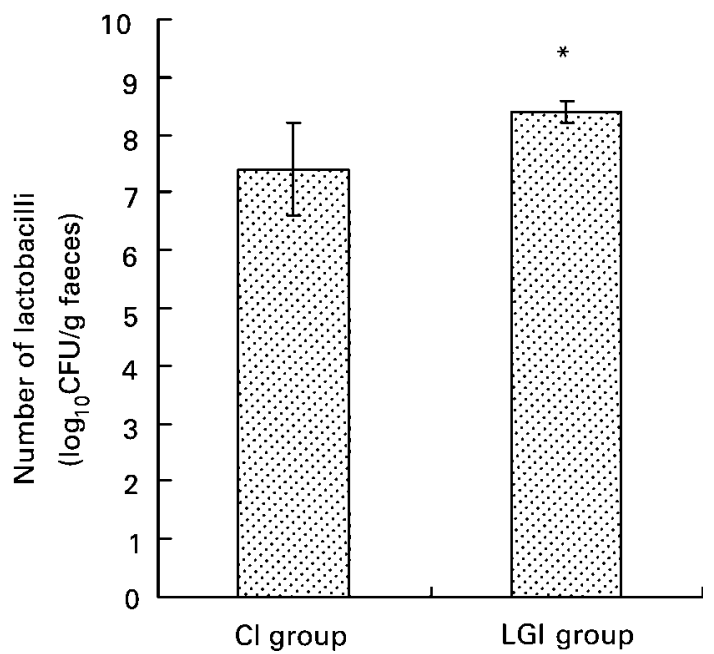

Fig. 4. Number of lactobacilli $\left(\log _{10}\right.$ colony-forming units $(C F U) / g$ wet weight faeces) of the mice in the Lactobacillus gasseri (LGI) group and the control $(\mathrm{Cl})$ group. Values are means, with their standard errors represented by vertical bars. *Mean value was significantly different from that for the $\mathrm{Cl}$ group $(P<0.05)$. higher concentration. The average equol concentration in the CI group was ten times higher compared with the LGI group (Fig. 5).

\section{Discussion}

Daidzein is a major component of the isoflavones, and it is metabolised to equol by the intestinal bacterial flora (Bowey et al. 2003). Both compounds have an oestrogenic effect and may contribute to protection against CVD. When used as a probiotic, L. gasseri can affect the intestinal flora and thus influence isoflavonoid metabolism in the digestive tract. In the present study we investigated the effects of L. gasseri on caecal and plasma isoflavonoids and caecal enzyme activity in mice.

Among mice that were administered L. gasseri (LGI group), the equol concentration in the plasma was significantly lower than in untreated mice (CI group). However, the plasma daidzein concentrations were significantly higher in the LGI group. This may be because high concentrations of daidzin (the glucoside of daidzein) were present in the soyabean extract included in the diet of the mice. It has been reported that absorption of daidzin is lower than daidzein in human subjects (Izumi et al. 2000). Setchell et al. (2002b) reported that daidzein was absorbed as the aglycone form after hydrolysis of the sugar moieties. There was a significant difference in the plasma equol: plasma daidzein ratio between the LGI and CI groups. The plasma equol:plasma daidzein ratio in the CI group was significantly higher compared with that in the LGI group. However, no significant difference in the ratio of the total amount of daidzein present as aglycone in the caecum to the total amounts of equol present as aglycone

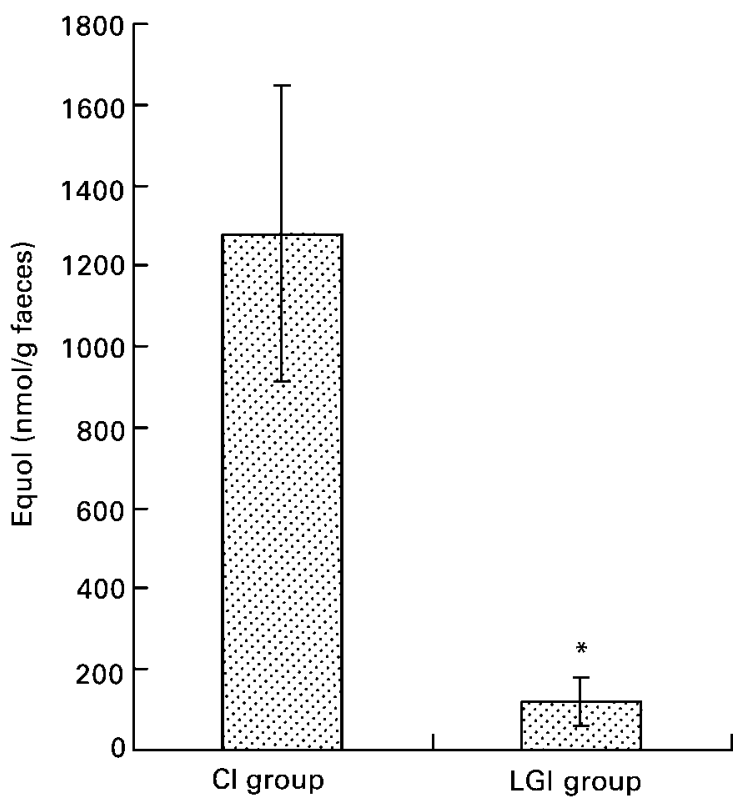

Fig. 5. Amounts of equol (nmol/g wet weight faeces) of the mice in the Lactobacillus gasseri (LGI) group and the control (Cl) group in an in vitro incubation of daidzin with the faecal flora of mice. Values are means, with their standard errors represented by vertical bars. *Mean value was significantly different from that for the $\mathrm{Cl}$ group $(P<0.05)$. 
in the caecum was observed between the LGI and CI groups. It has been reported that the slower plasma clearance of equol is striking compared with its precursor, daidzein (Setchell et al. 2002a). A significant difference of plasma clearance between equol and daidzein might contribute to the difference of the equol:daidzein ratio between the plasma and caecal contents.

The lactobacilli can survive long periods in mucin (Sims, 1964). Most lactobacilli have been shown to adhere via binding to collagen type I (Aleljung et al. 1991). We showed that the L. gasseri strain used in the present experiment possesses $\beta$-glucosidase activity in vitro (data not shown). $\beta$-Glucosidase from the $L$. gasseri in the upper gastrointestinal tract could contribute to the higher plasma daidzein concentrations found in the LGI group of mice by hydrolysing the daidzin to daidzein. No significant differences were observed in the present experiments in the caecal $\beta$-glucosidase and $\beta$-glucuronidase activities of the two groups. However, it has been reported that $L$. gasseri administration actually reduces faecal $\beta$-glucuronidase activities (Pedrosa et al. 1995) in human subjects. Tamura et al. (1996) reported that some strains of Bacteroides and Clostridium have $\beta$-glucosidase and $\beta$-glucuronidase activities in vitro. The inconsistent reports of $L$. gasseri effects on $\beta$-glucuronidase activity in the gastrointestinal tract might be due in part to differences in the compositions of human and mouse intestinal flora.

The numbers of lactobacilli were significantly greater in the LGI group than in the CI group. Saxelin et al. (1991) demonstrated that all human volunteers were colonized following the administration of $L$. casei strain GG after only $4 \mathrm{~d}$ of treatment. Lactobacilli shedding also increased after only $4 \mathrm{~d}$ of treatment in the present study (although we did not identify the lactobacilli in the mouse faeces to the species level).

The present study is the first to demonstrate the effects of $L$. gasseri on faecal floral equol production. Equol is a bacterial metabolite derived from daidzein (Bowey et al. 2003) and it has been reported that equol excretion in human subjects varies among individuals (Rowland et al. 2000). We found that in the CI group, which was not fed lactobacilli, both the plasma equol concentration and the total amount of equol present as aglycone in the caecal contents were significantly higher compared with those which were administered lactobacilli. When daidzein was incubated in vitro with mouse faecal flora, the concentrations of equol produced were again significantly higher in the CI group. This result suggests that equol production of the faecal flora was inherently greater in the CI group and that L. gasseri may suppress its production.

Several metabolic compounds produced by lactic acid bacteria such as organic acids, fatty acids, and $\mathrm{H}_{2} \mathrm{O}_{2}$ have antimicrobial effects (Ouwehand, 1998). Bacteriocins produced by some strains of lactic acid bacteria also have antimicrobial effects (Ouwehand, 1998). Increased numbers of lactobacilli would therefore affect the composition and/or metabolic activity of intestinal flora in the LGI mice. It has been reported that antibiotic treatments result in a marked reduction in plasma equol concentrations and altered plasma isoflavonoid patterns in cynomolgus monkeys (Macaca fascicularis; Blair et al. 2003). The alterations in the plasma isoflavonoid profile are unique to the antibiotic used. We also observed different plasma isoflavonoid profiles in the LGI and CI groups. Administration of L. gasseri could have caused the change of equol production by changing the gastrointestinal environment including the intestinal microflora. On the other hand Morotomi \& Mutai (1986) have investigated the in vitro binding to intestinal bacteria of amino acid pyrolysates. The administration of $L$. gasseri might also affect the binding capacity of intestinal flora for isoflavonoids. In summary, the present data suggest that the administration of L. gasseri influences the effect of isoflavonoids on the host, probably through changes in the gastrointestinal environment.

\section{References}

Aleljung P, Paulsson M, Emody L, Andersson M, Naidu AS \& Wadström T (1991) Collagen binding by lactobacilli. Curr Microbiol 23, 33-38.

Anthony MS, Clarkson TB \& Williams JK (1998) Effects of soy isoflavones on atherosclerosis: potential mechanisms. Am J Clin Nutr 68, 1390S-1393S.

Blair RM, Appt SE, Franke AA \& Clarkson TB (2003) Treatment with antibiotics reduces plasma equol concentration in cynomolgus monkeys (Macaca fascicularis). J Nutr 133, 2262-2267.

Bowey E, Adlercreutz H \& Rowland I (2003) Metabolism of isoflavones and lignans by the gut microflora: a study in germ-free and human flora associated rats. Food Chem Toxicol 41, $631-636$.

Cruz ML, Wong WW, Mimouni F, Hachey DL, Setchell KD, Klein PD \& Tsang RC (1994) Effects of infant nutrition on cholesterol synthesis rates. Pediatr Res 35, 135-140.

Fuller R (1989) A review: probiotics in man and animals. J Appl Bacteriol 66, 365-378.

Gilliland SE (1990) Health and nutritional benefits from lactic acid bacteria. FEMS Microbiol Rev 7, 175-188.

Hammes WP \& Tichaczek PS (1994) The potential of lactic acid bacteria for the production of safe and wholesome food. $Z$ Lebensm Unters Forsch 198, 193-201.

Isolauri E, Juntunen M, Rautanen T, Sillanaukee P \& Koivula T (1991) A human Lactobacillus strain (Lactobacillus casei sp strain GG) promotes recovery from acute diarrhea in children. Pediatrics 88, 90-97.

Izumi T, Piskula MK, Osawa S, Obata A, Tobe K, Saito M, Kataoka S, Kubota Y \& Kikuchi M (2000) Soy isoflavone aglycones are absorbed faster and in higher amounts than their glucosides in humans. J Nutr 130, 1695-1699.

Lampe JW, Karr SC, Hutchins AM \& Slavin JL (1998) Urinary equol excretion with a soy challenge: influence of habitual diet. Proc Soc Exp Biol Med 217, 335-339.

Lieberman S (1996) Are the differences between estradiol and other estrogens, naturally occurring or synthetic, merely semantical? J Clin Endocrinol Metab 81, 850-851.

Morotomi M \& Mutai M (1986) In vitro binding of potent mutagenic pyrolysates by intestinal bacteria. J Natl Cancer Inst 77, 195-201.

Ouwehand AC (1998) Antimicrobial components from lactic acid bacteria. In Lactic Acid Bacteria: Microbial Ecology and Functional Aspects, 2nd ed. pp. 139-159 [S Salminen and A von Wright, editors]. New York: Marcel Dekker Inc.

Pedrosa MC, Golner BB, Goldin BR, Barakat S, Dallal GE \& Russell RM (1995) Survival of yogurt-containing organisms and Lactobacillus gasseri $(\mathrm{ADH})$ and their effect on bacterial 
enzyme activity in the gastrointestinal tract of healthy and hypochlorhydric elderly subjects. Am J Clin Nutr 61, 353-359.

Reeves PG, Nielsen FH \& Fahey GC Jr (1993) AIN-93 purified diets for laboratory rodents: final report of the American Institute of Nutrition ad hoc writing committee on the reformulation of the AIN-76A rodent diet. J Nutr 123, 1939-1951.

Rowland IR, Mallett AK \& Wise A (1983) A comparison of the activity of five microbial enzymes in cecal content from rats, mice, and hamsters, and response to dietary pectin. Toxicol Appl Pharmacol 69, 143-148.

Rowland IR, Wiseman H, Sanders TA, Adlercreutz H \& Bowey EA (2000) Interindividual variation in metabolism of soy isoflavones and lignans: influence of habitual diet on equol production by the gut microflora. Nutr Cancer 36, 27-32.

Salminen E, Elomaa I, Minkkinen J, Vapaatalo H \& Salminen S (1988) Preservation of intestinal integrity during radiotherapy using live Lactobacillus acidophilus cultures. Clin Radiol 39, 435-437.

Salminen S, Isolauri E \& Salminen E (1996) Clinical uses of probiotics for stabilizing the gut mucosal barrier: successful strains and future challenges. Antonie Van Leeuwenhoek 70, 347-358.

Saxelin M, Elo S, Salminen S \& Vapaatalo H (1991) Dose response colonization of faeces after oral administration of Lactobacillus casei strain GG. Microbiol Ecol Health Dis 4, 209-214.
Setchell KD, Borriello SP, Hulme P, Kirk DN \& Axelson M (1984) Nonsteroidal estrogens of dietary origin: possible roles in hormone-dependent disease. Am J Clin Nutr 40, 569-578.

Setchell KD, Brown NM \& Lydeking-Olsen E (2002a) The clinical importance of the metabolite equol - a clue to the effectiveness of soy and its isoflavones. J Nutr 132, 3577-3584.

Setchell KD, Brown NM, Zimmer-Nechemias L, Brashear WT, Wolfe BE, Kirschner AS \& Heubi JE (2002b) Evidence for lack of absorption of soy isoflavone glycosides in humans, supporting the crucial role of intestinal metabolism for bioavailability. Am J Clin Nutr 76, 447-453.

Setchell KD, Zimmer-Nechemias L, Cai J \& Heubi JE (1997) Exposure of infants to phyto-oestrogens from soy-based infant formula. Lancet 350, 23-27.

Shutt DA \& Cox RI (1972) Steroid and phyto-oestrogen binding to sheep uterine receptors in vitro. J Endocrinol 52, 299-310.

Sims W (1964) The effect of mucin on the survival of lactobacilli and streptococci. J Gen Microbiol 37, 335-340.

Tamura M, Hirayama K \& Itoh K (1996) Comparison of colonic bacterial enzymes in gnotobiotic mice monoassociated with different intestinal bacteria. Microbiol Ecol Health Dis 9, 287-294.

Zhu WM, Liu W \& Wu DQ (2000) Isolation and characterization of a new bacteriocin from Lactobacillus gasseri KT7. J Appl Microbiol 88, 877-886. 